Canad. Math. Bull. Vol. 20 (3), 1977

\title{
ORTHOGONALLY COMPLETE RINGS
}

\author{
BY \\ R. RAPHAEL AND W. STEPHENSON
}

Introduction. In this note we continue the study of Abian's order for reduced rings initiated in papers such as [1], [5], [3], [4]. A simple proof is given of Abian's result that taking suprema commutes with ring multiplication. The properties of orthogonally complete rings and of rings satisfying chain conditions with respect to Abian's order are investigated. Finally those rings $\boldsymbol{R}$ for which $R[x]$ and $R[[x]]$ are orthegonally complete are characterized. These results provide interesting examples and counterexamples in the study of Abian's order relation.

Terminology. A ring $R$ is said to be reduced if it contains no non-zero nilpotent elements. Throughout this note we consider only reduced rings with a 1 , unless otherwise stated. We recall some elementary facts about such rings. If $a, b \in R$ and $a b=0$, then $b a=0$. Hence the left and right annihilators of any subset $S \subseteq R$ form a two-sided ideal which we denote by $\operatorname{Ann}(S)$. In particular for any $a \in R, \operatorname{Ann}(a) \cap R a=0$. For other standard terminology the reader is referred to [7].

$\$ 1$. Any reduced ring can be written as a subdirect product of integral domains (not necessarily commutative). Since any integral domain can be ordered by decreeing that $c \geq 0$ for any $c$, this induces an ordering on $R$ given by $a \leq b$ if $a^{2}=a b$ (see [1] and [5] for details). With respect to this ordering we can discuss upper and lower bounds, suprema and infima of subsets of $R$ in the usual manner.

1.1. Proposition. Let $X$ be a subset of a ring $R$. Then the following are equivalent for any $c \in R$.

(i) $c=\sup (X)$.

(ii) $c$ is an upper bound for $X$ and $\operatorname{Ann}(X) \subseteq \operatorname{Ann}(c)$.

In fact $\operatorname{Ann}(X)=\operatorname{Ann}(c)$.

Proof. (ii) $\Rightarrow$ (i). Let $d$ be an upper bound for $X$. Then for all $x \in X, x \leq c$ and $x \leq d$ and so $x c=x^{2}=x d$. Thus $c-d \in \operatorname{Ann}(X) \subseteq \operatorname{Ann}(c)$ and so $(c-d) c=0$. Hence $c \leq d$ and $c=\sup (X)$.

(i) $\Rightarrow$ (ii). Suppose that $c=\sup (X)$. Then $c$ is an upper bound for $X$. Let $d \in \operatorname{Ann}(X)$. Then for all $x \in X, x^{2}=x c=x(c+d)$, and so $c+d$ is an upper

Received by the editors Feb. 1, 1974 and, in revised form, Aug. 26, 1976. 
bound for $X$. Therefore $c \leq(c+d)$ and $c^{2}=c(c+d)$. Thus $c d=d c=0$ and $\operatorname{Ann}(X) \subset \operatorname{Ann}(c)$.

If $c$ is an upper bound for $X$, then $x^{2}=x c$ for all $x \in X$. Hence $\operatorname{Ann}(c) \subseteq$ $\operatorname{Ann}\left(x^{2}\right)=\operatorname{Ann}(x)$ for all $x \in X$, and so $\operatorname{Ann}(c) \subseteq \operatorname{Ann}(X)$, giving $\operatorname{Ann}(X)=$ $\operatorname{Ann}(c)$.

As a corollary we give a simple proof of a result of Abian (see Lemma 8 of [1]).

1.2. Corollary. Suppose that $X$ is a subset of a ring $R$ such that $\sup (X)$ exists. Then for every $a \in R, \sup (a X)$ exists and equals $a \sup (X)$.

Proof. Let $b=\sup (X)$. It is easily seen that $a b$ is an upper bound for $a X$. By 1.1. $\operatorname{Ann}(X) \subseteq A n n(b)$, and so $\operatorname{Ann}(a X) \subseteq \operatorname{Ann}(a b)$. By 1.1. $\sup (a X)$ exists and $\sup (a X)=a b=a \sup (X)$.

REMARK. If $X$ is a subset of.$R$ containing elements $c$ and $d$ such that $c d=0$, then $X$ has an infimum, namely 0 . For let $b$ be a lower bound for $X$. Then $b^{2}=b c=b d$ and $b^{2} d=b c d=0$. Hence $b d=0$ and $b^{2}=0$. Therefore $b=0$ and $\inf (X)=0$. For such a set $X$, it is clear that for any $a \in R, \inf (a X)=$ $a \inf (X)=0$.

However, in general 1.2 is not true if 'sup' is replaced by 'inf'. Let $R=\{(a, b) \in Z \times Z: a \equiv b \bmod 2\}$. Let $X=\left\{x_{1}, x_{2}\right\}$, where $x_{1}=(1,1)$ and $x_{2}=$ $(1,3)$. It is easily seen that $\inf (X)=(0,0)$. If $a=(2,0)$, then $a X=\{(2,0)\}$ and $\inf (a X)=(2,0) \neq(0,0)=a \inf (X)$.

$\S 2$. It is easy to see that for a ring $R, R$ is bounded above if and only if $R$ is a Boolean ring. Thus to obtain interesting completeness results for $(R, \leq)$ we have to consider weaker forms of completeness.

A set $X$ of non-zero elements of a ring $R$ is said to be orthogonal if $x y=0$ for all $x, y \in X$ such that $x \neq y$. We note that if $X$ is orthogonal, then $\sum_{x \in X} R x$ is a direct sum. A ring $R$ is said to be orthogonally complete if every orthogonal set in $R$ has a supremum. The reader is referred to [5], [3], [4], [8] for details about such rings. We note that the remark following 1.2 shows that any orthogonal set always has an infimum.

2.1. Lemma. Let I be a non-zero left ideal of a ring $R$. If $\left\{a_{\alpha}\right\}_{\mathrm{A}}$ is a maximal orthogonal set of elements of $I$ then $\operatorname{Ann}(I)=\operatorname{Ann}\left(\left\{a_{\alpha}\right\}_{A}\right)$.

Proof. Clearly $\operatorname{Ann}(I) \subseteq \operatorname{Ann}\left(\left\{a_{\alpha}\right\}_{A}\right)$. Suppose that $0 \neq c \in I \cap \operatorname{Ann}\left(\left\{a_{\alpha}\right\}_{A}\right)$. Then $\left\{a_{\alpha}\right\}_{A} \cup\{c\}$ is orthogonal, contradicting the choice of $\left\{a_{\alpha}\right\}_{A}$. Thus $I \cap$ $\operatorname{Ann}\left(\left\{a_{\alpha}\right\}_{A}\right)=0$ and $\operatorname{Ann}\left(\left\{a_{\alpha}\right\}_{A}\right) \subseteq \operatorname{Ann}(I)$, giving the result.

Our next result gives some necessary conditions for a ring to be orthogonally complete. The general problem raised in [3] of giving an "element-wise" characterization of such rings remains open. 
2.2. Proposition. Let $R$ be orthogonally complete

(i) For every non-empty set $X \subseteq R$, there exists $a \in R$ such that $\operatorname{Ann}(X)=$ $\operatorname{Ann}(a)$. Moreover, if $\operatorname{Ann}(X) \subseteq \operatorname{Ann}(b)$ for some $b \in R$, then a can be chosen so that $b \leq a$.

(ii) For every $b \in R$, there exists a regular element $c \in R$, that is $\operatorname{Ann}(c)=0$, such that $b \leq c$.

Proof. (i) If $\operatorname{Ann}(X) \subseteq \operatorname{Ann}(b)$, then $R b \subseteq \operatorname{Ann} \operatorname{Ann}(b) \subseteq \operatorname{Ann} \operatorname{Ann}(X)$. Without loss of generality we may assume that $b \neq 0$ and by Zorn's lemma we can choose a maximal orthogonal set $\left\{a_{\alpha}\right\}_{A}$ of elements of Ann Ann(X) containing $b$. Let $a=\sup \left(\left\{a_{\alpha}\right\}_{\mathrm{A}}\right)$ and note that $b \leq a$. By 1.1 and 2.1, $\operatorname{Ann}(a)=$ $\operatorname{Ann}\left(\left\{a_{\alpha}\right\}_{A}\right)=\operatorname{Ann} \operatorname{Ann} \operatorname{Ann}(X)=\operatorname{Ann}(X)$.

(ii) Let $X=R$ in (i).

Remark. In any ring $R$, regular elements are always maximal in $(R, \leq)$. 2.2(ii) shows that in an orthogonally complete ring the regular elements are the only maximal elements and moreover that every element of $R$ is always less than or equal to such an element.

EXAMPLE. Any strongly regular Baer ring $R$ satisfies the conditions of 2.2 but is orthogonally complete if and only if $R$ is a self-injective ring. The example given in [9] (see Example 3) shows that this is not necessarily the case and so that the conditions in 2.2 are not sufficient to ensure that $R$ is orthogonally complete.

Our next result extends Proposition 8 of [3].

2.3. The following are equivalent for a ring $R$.

(i) $(R, \leq)$ satisfies the ascending chain condition.

(ii) $(R, \leq)$ satisfies the descending chain condition and $R$ is orthogonally complete.

(iii) Any orthogonal set in $R$ is finite.

(iv) $R$ satisfies the ascending (descending) chain condition on annihilators.

Proof. The equivalences (i) $\Leftrightarrow$ (ii) $\Leftrightarrow$ (iii) are immediate from the following observations:

(a) if $\left\{a_{i}\right\}$ is a strictly ascending or descending chain, then $\left\{a_{i}-a_{i+1}\right\}$ is an orthogonal set,

(b) if $\left\{a_{i}\right\}$ is a finite orthogonal set, then $\sup \left\{a_{i}\right\}$ exists and equals $\sum a_{i}$,

(c) if $\left\{a_{i}\right\}$ is an orthogonal set, then $\left\{\sup \left\{a_{i}: 1 \leq i \leq j\right\}\right\}$ is a strictly ascending chain and $\left\{\sup \left\{a_{j}: j \geq i\right\}\right\}$ is a strictly descending chain (provided that suprema exist).

The equivalence (iii) $\Leftrightarrow$ (iv) follows from the following observations:

(d) since annihilators are two-sided ideals the descending and ascending chain conditions on these ideals are equivalent, 
(e) if $\left\{a_{i}\right\}$ is an orthogonal set, then $\left\{\operatorname{Ann}\left\{a_{1}, \ldots, a_{i}\right\}\right\}$ is a strictly descending chain of annihilators,

(f) if $\left\{I_{i}\right\}$ is a strictly ascending chain of annihilators, then $I_{i+1} \cap \operatorname{Ann}\left(I_{i}\right) \neq(0)$ and if $0 \neq a_{i} \in I_{i+1} \cap \operatorname{Ann}\left(I_{i}\right)$, then $\left\{a_{i}\right\}$ is an orthogonal set.

EXANiPLE. It is possible for $(R, \leq)$ to have the descending chain condition without having the ascending chain condition. For let $K$ be a field. Define $R$ to be $K\left[x_{1}, x_{2}, x_{3}, \ldots\right]$ with the relations $x_{i} x_{j}=0$ for $i \neq j . R$ is a subdirect product of the polynomial rings $K\left[x_{i}\right]$ and so is reduced. Using this subdirect product representation it is not difficult to show that for any $g \in R$ there are only a finite number of $f \in R$ such that $f \leq g$.

Thus $(R, \leq)$ has the descending chain condition. On the other hand the set $\left\{x_{i}\right\}_{1}^{\infty}$ is orthogonal and so, by $2.3,(R, \leq)$ does not have the ascending chain condition.

$\S 3$. In this section we consider when the polynomial and power series rings $R[x]$ and $R[[x]]$ are orthogonally complete. First we need the following result.

3.1. Lemma. Let $f=\sum_{0}^{\infty} a_{i} x^{i}, g=\sum_{0}^{\infty} b_{i} x^{i}$ belong to $R[[x]]$. Then $f g=0$ if and only if $a_{i} b_{j}=0$ for all $i \geq 0$ and $j \geq 0$.

Proof. See Armendariz [2].

3.2. Proposition. The following are equivalent for a ring $R$.

(i) $R[x]$ is orthogonally complete.

(ii) $R[x]$ satisfies (i)-(iv) of 2.3 .

(iii) $R$ satisfies (i)-(iv) of 2.3 .

Proof. (ii) $\Rightarrow$ (i) is clear.

(i) $\Rightarrow$ (iii). Suppose that $\left\{a_{1}, a_{2}, \ldots\right\}$ is an infinite urthogonal set in $R$. Then the infinite orthogonal set $\left\{a_{1} x, a_{2} x^{2}, \ldots\right\}$ has no supremum in $R[x]$, contradicting (i).

(iii) $\Rightarrow$ (ii). Suppose that $\left\{f_{\alpha}\right\}_{A}$ is an infinite orthogonal set in $R[x]$. By 3.1, the set $\left\{a_{\alpha}\right\}_{\mathrm{A}}$ of leading coefficients of the $f_{\alpha}$ 's is an infinite orthogonal set of $R$, contradicting (iii).

Remark. It can easily be shown that 3.2 holds for any polynomial ring over any number of commuting indeterminates finite or infinite.

3.3. Proposition. The following are equivalent for $a$ ring $\boldsymbol{R}$.

(i) $R[[x]]$ is orthogonally complete.

(ii) $R$ is orthogonally complete.

Proof. (i) $\Rightarrow$ (ii). Suppose that $\left\{a_{\alpha}\right\}_{A}$ is an orthogonal set in $R$. By hypothesis, $f=\sup \left(\left\{a_{\alpha}\right\}_{A}\right)$ exists in $R[[x]]$. If $f=\sum_{0}^{\infty} b_{i} x^{1}$, we have $a_{\alpha}^{2}=a_{\alpha} f$ for all $\alpha \in A$ and so $a_{\alpha} b_{i}=0$, for all $\alpha$ and $i>0$. By 1.1, Ann $\left(\left\{a_{\alpha}\right\}_{A}\right) \subseteq \operatorname{Ann}(f)$ and so $b_{i} f=0$ for 
all $i>0$. Hence $b_{i}^{2}=0$ and $b_{i}=0$ for all $i>0$ and so $f=\sup \left(\left\{a_{\alpha}\right\}\right)$ is in $R$ and $R$ is orthogonally complete.

(ii) $\Rightarrow$ (i). Suppose that $\left\{f_{\alpha}\right\}_{A}$ is an orthogonal set in $R[[x]]$, where $f_{\alpha}=$ $\sum_{i=0}^{\infty} a_{\alpha i} x^{i}$. By $3.1 a_{\alpha i} a_{\beta j}=0$ for all $i, j$ if $\alpha \neq \beta$. Hence for each $i,\left\{a_{\alpha i}\right\}_{A}$ is an orthogonal set in $R$. Let $b_{i}=\sup \left(\left\{a_{\alpha i}\right\}_{A}\right)$, which exists by (ii), and define $f=\sum_{0}^{\infty} b_{i} x^{i}$. The coefficient of $x^{k}$ in $f_{\alpha} f$ is $\sum_{i+j=k} a_{\alpha i} b_{j}$. By $1.2 a_{\alpha i} b_{j}=$ $a_{\alpha i} \sup \left(\left\{a_{\beta j}\right\}_{A}\right)=\sup \left(\left\{a_{\alpha i} a_{\beta j}\right\}_{A}\right)=a_{\alpha i} a_{\alpha j}$. Thus the coefficients of $x^{k}$ in $f_{\alpha} f$ and $f_{\alpha}^{2}$ are equal and $f_{\alpha}^{2}=f_{\alpha} f$.

Now suppose that $g f_{\alpha}=0$ for all $\alpha$ where $g=\sum_{0}^{\infty} c_{i} x^{i}$. By 3.1, $c_{i} a_{\alpha j}=0$ for all $\alpha$, $i$, and $j$. Now $c_{i} b_{j}=c_{i} \sup \left(\left\{a_{\alpha j}\right\}_{A}\right)=\sup \left(\left\{c_{i} a_{\alpha j}\right\}_{A}\right)=0$. Thus $g f=0$ and $f=$ $\sup \left(\left\{f_{\alpha}\right\}_{A}\right)$ by 1.1 .

REMARK. 3.3 can be shown to hold for power series rings in any finite number of indeterminates. For an infinite number of indeterminates 3.3 may or may not hold depending on which definition of the power series is adopted. (For possible definitions see, for example, [6].)

ACKNOWLEDgEMENTS. The authors wish to acknowledge the contribution made by W. Burgess to the results presented in this note. The first author was supported by the National Research Council of Canada (A 7752) and the Canada Council (W 750463). The second author was partially supported by the National Research Council of Canada through Professor J. Lambek.

\section{BIBLIOGRAPHY}

1. A. Abian, Direct product decomposition of commutative semi-simple rings, Proc. Amer. Math. Soc. 24 (1970), 502-507.

2. E. P. Armendariz, A note on extensions of Baer and p.p.-rings, J. Austral. Math. Soc. 18 (1974), 470-473.

3. W. D. Burgess and R. Raphael, Abian's order relation and orthogonal completeness for reduced rings, Pac. J. Math. 54 (1974), 55-64.

4. W. D. Burgess and R. Raphael, Complete and orthogonally complete rings, Can. Jour. Math. 27 (1975), (to appear).

5. M. Chacron, Direct products of division rings and a paper of Abian. Proc. Amer. Math. Soc. 29 (1971), 259-262.

6. R. W. Gilmer, Power series rings over a Krull domain, Pac. Jour. Math. 29 (1969), 543-549.

7. J. Lambek, Lectures on rings and modules, Waltham, Massachusetts, Ginn, 1966.

8. T. Speed, A note on commutative Baer rings, J. Australian, Math. Soc. 14 (1972), 257-263.

9. Y. Utumi, On continuous regular rings and semi-simple self-injective rings, Can. J. Math. 12 (1960), 597-605.

DEPARTMENT OF MATHEMATICS,

Concordia University, Montreal, CANada

on leave at Département de Mathématioues,

Université de Poitiers, Poitiers, France

Department of Mathematics,

MCGILl UNIVERSITY, MONTREAL,

PERMANENT ADDRESS:

Department of Mathematics,

BedFord College,

Regent's PARK,

LONDON, NW1 4NS, ENGLAND. 